\title{
Tronco Hepato-Espleno-Mesentérico. Descripción de una Disposición Anómala del Tronco Celíaco
}

\author{
Hepato-Splenic-Mesenteric Trunk. Description of an Abnormal Disposition of the Celiac Trunk
}

\author{
Mariano del Sol ${ }^{1,2}$; Nicolás Ernesto Ottone ${ }^{1,3}$ \& Bélgica Vásquez ${ }^{4}$
}

\begin{abstract}
DEL SOL, M.; OTTONE, N. E. \& VÁSQUEZ, B. Tronco hepato-espleno-mesentérico. Descripción de una disposición anómala del tronco celíaco. Int. J. Morphol., 36(4):1525-1528, 2018.

RESUMEN: El estudio de los patrones arteriales de distribución vascular requiere un conocimiento preciso de las variaciones anatómicas, tanto de origen y distribución de las arterias, como de sus correspondientes ramas arteriales. En este trabajo se describe la presencia de un tronco hepato-espleno-mesentérico, disposición arterial altamente infrecuente. El conocimiento de las distintas posibilidades de disposición de las arterias correspondientes al tronco celíaco y sus ramas será de importancia para la interpretación adecuada de estudios imagenológicos, como así también para la planificación precisa de actos quirúrgicos e intervencionistas en la región abdominal.
\end{abstract}

PALABRAS CLAVE: Anatomía Tronco hepato-espleno-mesentérico; Variación anatómica; Tronco celíaco.

\section{INTRODUCTION}

El estudio preciso de la vascularización abdominal ha sido de gran interés desde hace muchísimos años, comenzando con los estudios de Haller en 1756, Tidemann en 1822, y el siglo pasado, con la destacada investigación de Adachi en 1928. En la actualidad, la aplicación del conocimiento de la disposición normal y variable de las arterias abdominales resultó de fundamental importancia para cirujanos y radiológos al momento de planificar procedimientos quirúrgicos y radiológicos que involucren dichas arterias. En este sentido, las variaciones en la conformación del tronco celíaco y la distribución de sus ramas puede determinar la presencia de disposiciones variables que constituyen un desafío su abordaje e intervención. Son destacados los trabajos clásicos de Michels (1966) y Vandamme et al. (1969) en relación a las variaciones del tronco celíaco y arterias hepáticas, y más cercano a este tiempo, las investigaciones a través de angiografías digitales realizadas por Hiatt et al. (1994). Normalmente el tronco celiaco es el primer ramo visceral que nace de la parte de anterior de la parte abdominal de la aorta inmediatamente abajo del hiato aórtico del diafragma a nivel de los cuerpos vertebrales de T12 o L1. El tronco celiaco se divide en las arterias gástrica izquierda, hepática común y esplénica. Por otra parte, el tronco celiaco puede tener un origen común con la arteria mesentérica su- perior, la cual normalmente se origina de la parte anterior de la parte abdominal de la aorta, aproximadamente $1 \mathrm{~cm}$ distal al tronco celiaco.

Se ha señalado que el hecho más constante del tronco celiaco es la variedad de sus ramas y los trayectos por lo que la sangre alcanza los órganos que irriga, sin embargo la presencia de un tronco hepato-esplénico-mesentérico no es una variación común.

El objetivo del presente trabajo consistió en dar a conocer el hallazgo de un caso de ausencia de la disposición normal del tronco celíaco, reemplazado por la presencia un tronco hepato-espleno-mesentérico. El conocimiento de esta disposición variable del tronco celíaco será crucial para afrontar intervenciones clínicas, quirúrgicas e imagenológicas de dicha región.

\section{REPORTE DE CASO}

Durante una disección de rutina en un cadáver adulto, masculino, caucásico, fijado y conservado con una solu-

\footnotetext{
${ }^{1}$ Programa de Doctorado en Ciencias Morfológicas, Facultad de Medicina, Universidad de La Frontera, Temuco, Chile.

${ }^{2}$ Center of Excellence in Morphological and Surgical Studies (CEMyQ), Universidad de La Frontera, Temuco, Chile.

${ }^{3}$ Centro de Investigación en Ciencias Odontológicas (CICO), Facultad de Odontología, Universidad de La Frontera, Temuco, Chile.

${ }^{4}$ Facultad de Ciencias de la Salud, Universidad de Tarapacá, Arica, Chile.
} 
ción de formalina al $10 \%$, se llevó a cabo el estudio de la región celíaca. Luego de proceder a la apertura de la pared abdominal mediante una incisión en boca de horno, se seccionaron los músculos de las paredes anterolaterales del abdomen, logrando acceder al compartimiento supramesocólico, con el objetivo de identificar los elementos vasculares de la región.

$\mathrm{Al}$ realizar la disección, se identificó la ausencia de un tronco celíaco clásico, formado por las arterias hepática común, esplénica y gástrica izquierda, y en su reemplazo se presentó un tronco hepato-espleno-mesentérico (THEM). El THEM dio origen a las arterias hepática común, esplénica y mesentérica superior. La arteria hepática común, daba origen a la arteria hepática izquierda, la cual antes de alcanzar el hilio hepático, proporcionaba la arteria gástrica izquierda. La arteria esplénica dio origen a la arteria gastroduodenal. Por otra parte, desde la arteria mesentérica superior se originaba la arteria hepática derecha (Fig. 1).

\section{DISCUSIÓN}

El tronco celiaco es el primer ramo visceral que se origina bajo el arco aórtico a nivel de los cuerpos vertebrales de T12 a L1. Es normalmente bastante corto, midiendo entre 1,5 a $2 \mathrm{~cm}$ de longitud. Por otra parte, la arteria mesentérica superior se origina distal al tronco celiaco entre 1 a $1,5 \mathrm{~cm}$ de éste y, por tanto, a nivel de L1 - L2. El tronco celíaco proporciona clásicamente, como ramas terminales, las arterias hepática común, esplénica y gástrica izquierda y

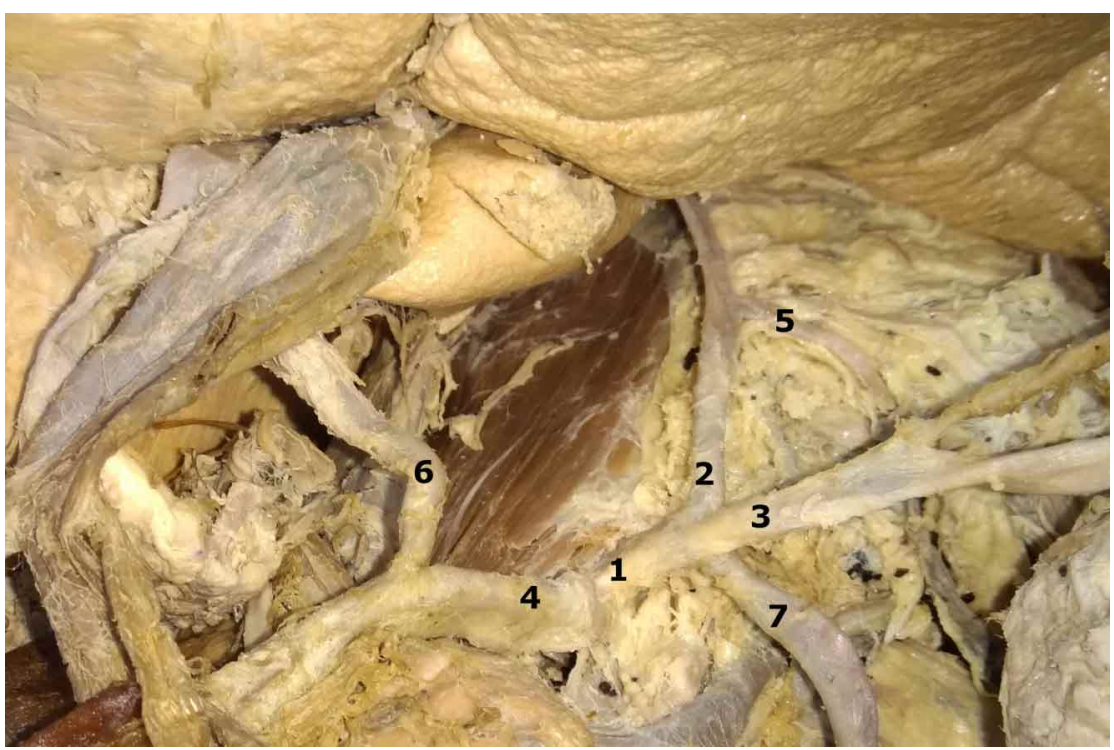

Fig. 1. 1. Tronco hepato-espleno-mesentérico; 2. Arteria hepática izquierda; 3. Arteria esplénica; 4. Arteria mesentérica superior; 5. Arteria gástrica izquierda; 6. Arteria hepática derecha; 7. Arteria gastroomental izquierda. podría, por tanto, ser denominado tronco gastrohepatoesplénico.

Bergman et al. (2006) describen la disposición normal del tronco celiaco en el $86 \%$ de 756 casos; Hiatt et al. hallaron la disposición clásica en 75,7 \% de los casos, mientras que Varotti et al. (2004) lo hizo en el 70,8 \%. MarcoClement et al. (2016) encontraron la disposición clásica en el 57,6 \% de los casos. Esto correspondería a una dispoción de tronco celíaco completo.

La variaciones arteriales tienen un correlato embriológico. Tandler (1906 apud Yi et al., 2007) demostró la existencia de una anastomosis longitudinal anterior entre las arterias intestinales metaméricas primitivas en embriones humanos. Las arterias hepática común, esplénica y gástrica izquierda se originan de esta anastomosis longitudinal. De esta manera, la desaparición y persistencia de porciones de dicha anastomosis determinaría la presencia en el adulto de variaciones anatómicas vasculares, en este caso, del tronco celíaco. Por lo tanto, el tronco celíaco y las arterias mesentéricas superior e inferior pueden estar unidos entre sí por esta anastomosis longitudinal continua, de persistencia embriológica.

El tronco celíaco puede proveer en forma variable más de tres ramas. Este ramo adicional puede ser una de las arterias frénicas inferiores, un tronco común para las arterias frénicas inferiores o un tronco común para las arterias frénica inferior y gástrica izquierda. También puede corresponder a la arteria gastroduodenal, una arteria esplénica accesoria, arteria cólica media, o una la arteria dorsal del páncreas.

En relación entonces a las variaciones arteriales, se describe la posibilidad de un tronco celíaco incompleto. En nuestro trabajo presentamos una disposición muy poco frecuente de ausencia de tronco celíaco, demostrando un tronco arterial conformado por las arterias hepática izquierda, esplénica y mesentérica superior, conformando el tronco hepato-espleno-mesentérico. Esta disposición es altamente infrecuente. Marco-Clement et al. identificaron la disposición del tronco celíaco a partir de disección en 43 cadáveres y por medio de imágenes de tomografía computada (TC) en 596 pacientes, estableciendo una clasificación, en la cual el tipo IIa correspondió a un tronco hepatoesplénico ( $11,6 \%$ de presencia en cadáveres; $4 \%$ 
de presencia en TC). Pero no encontraron la disposición descrita en este trabajo. En este sentido, Poynter (1922) describe la formación de un tronco celíaco-mesentérico en el 1,4\% de los casos. Por su parte, Panagouli et al. (2013) realizaron una revisión sistemática y encontraron el tronco hepato-espleno-mesentérico en 49 de un total de 12.196 casos, es decir, en el 0,40\% de los casos estudiados. Según Goss (1976) en raros casos el tronco celiaco y la arteria mesentérica superior están unidos.

Según Michels (1953) en el 3,5\% de los casos desde la aorta se origina un troncohepatoesplénico; la gástrica izquierda se origina independientemente de la aorta o en las arterias hepática o esplénica, en el 5,5\% un tronco gastroesplénico de donde se originan las arterias gástrica izquierda y la esplénica; la arteria hepática se origina de la aorta o en la arteria mesentérica superior; un tronco hepatogástrico en el 1,5\% de donde se originan la arteria gastrica izquierda y la arteria hepática; la arteria esplénica se origina de la aorta o en la arteria mesentérica superior.

El conocimiento de las variaciones a nivel del tronco celíaco y sus ramas es de fundamental importancia al momento de planificar cirugías, métodos diagnósticos y procedimientos invasivos de la región abdominal (del Sol et al., 2001; Hardy \& Jones, 2001). La presencia de la arteria hepática izquierda en el omento menor, producto de la variación descrita en este artículo, debe ser cuidadosamente identificada, por ejemplo, al momento de realizar una gastrectomía subtotal radical de carcinoma gástrico (Suzuki et al., 1971), debido a que se corre el riesgo de confundirla con una arteria gástrica izquierda, mucho más en este caso que ésta última se origina de la arteria hepática izquierda, pudiéndola ligar durante el acto quirúrgico, y generar una necrosis hepática izquierda. En este mismo sentido Goss señaló que la es la arteria gástrica izquierda quien proporciona la arteria hepática izquierda.

Por otro parte, en nuestro caso, la arteria hepática derecha está naciendo de la arteria mesentérica superior, aunque esta variación de origen es la más habitual. Esta variación debe ser considerada durante la colecistectomía y la pancreatectomía, ya que la arteria hepática derecha se encuentra ubicada entre la vena cava inferior y la vena porta antes de ubicarse a nivel del trígono cistohepático (Ottone et al., 2006). Todas estas variaciones de las arterias hepáticas deben considerarse e informarse en la planificación de un transplante hepático, ya que se bien las variaciones no están contraindicadas para la realización de la cirugía, su identificación permite disminuir los efectos colaterales y desarrollar una cirugía exitosa (Lee $e t$ al., 2003; Varotti et al.).
La identificación precisa de la disposición de las arterias abdominales, y en este caso del tronco celíaco, será de primordial importancia para una correcta planificación imagenológica y quirúrgica de la región abdominal.

DEL SOL, M.; OTTONE, N. E. \& VÁSQUEZ, B. Hepatosplenic-mesenteric trunk. description of an abnormal disposition of the celiac trunk. Int. J. Morphol., 36(4):1525$1528,2018$.

SUMMARY: The study of arterial patterns of vascular distribution requires a precise knowledge of the anatomical variations of both origin and distribution of the corresponding arteries and arterial branches. In this work, the presence of a hepatosplenic-mesenteric trunk is described, a highly infrequent arterial disposition. The knowledge of the different possibilities of disposition of the arteries corresponding to the celiac trunk and its branches will be of importance for the adequate interpretation of imaging studies, as well as for the precise planning of surgical and interventional acts in the abdominal region.

KEY WORDS: Hepato-splenic-mesenteric trunk; Anatomical variation; Celiac trunk.

\section{REFERENCIAS BIBLIOGRÁFICAS}

Adachi, B. Das Arteriensystem der Japaner. Kyoto, Universität zu Kyoto, 1928. V.2.

Bergman, R. A.; Afifi, A. K. \& Miyauchi, R. Celiac Trunk Arteries. Illustrated Encyclopedia of Human Anatomic Variation: Opus II: Cardiovascular System: Arteries: Abdomen. 2006. Disponible en: http:/ /www.anatomyatlases.org/AnatomicVariants/Cardiovascular/T ext/ Arteries/CeliacTrunk.shtml

del Sol, M.; Olave, E. \& Epulef, V. Variación anatómica de las arterias hepática propia y pancreaticoduodenal inferior. Aspectos anatomo-clínicos. Rev. Chil. Cs. Méd. Biol., 11(2):51-54, 2001.

Goss, C. M. Gray Anatomía. Salvat, Barcelona, 1976.

Haller, A. 1756 apud Vandamme, J. P. L. \& Bonte, J. op cit. The branches of the celiac trunk. Acta Anat., 122:110-4, 1985.

Hardy, K. J.; Jones, R.M. The hepatic artery: a reminder of surgical anatomy. J. R. Coll. Surg. Edinb., 46(3):168-70, 2001.

Hiatt, J. R.; Gabbay, J. \& Busuttil, R. W. Surgical anatomy of the hepatic arteries in 1000 cases. Ann. Surg., 220(1):50-2, 1994.

Lee, S. S.; Kim. T. K.; Byun, J. H.; Ha, H. K.; Kim. P. N.; Kim. A. Y.; Lee, S. G. \& Lee, M. G. Hepatic arteries in potential donors for living related liver transplantation: Evaluation eith multi-detector row ct angiography. Radiology, 227(2):391-9, 2003.

Marco-Clement, I.; Martinez-Barco, A.; Ahumada, N.; Simon, C.; Valderrama, J. M.; Sanudo, J. \& Arrazola, J. Anatomical variations of the celiac trunk: cadaveric and radiological study. Surg. Radiol. Anat., 38(4):501-10, 2016.

Michels, N. A. Variational anatomy of yhe hepatic, cystic and retroduodenal arteries. A statistical analysis of their origin, distribution and relations to the biliary ducts in two hundred bodies. AMA Arch. Surg., 66(1):20-34, 1953.

Michels, N. A. Newer anatomy of the liver and its variant blood supply and collateral circulation. Am. J. Surg., 112(3):337-47, 1966.

Ottone, N. E.; Arrotea, A.; Dominguez, M. L.; Lo Tártaro, M.; García de 
DEL SOL, M.; OTTONE, N. E. \& VÁSQUEZ, B. Tronco hepato-espleno-mesentérico. Descripción de una disposición anómala del tronco celíaco. Int. J. Morphol., 36(4):1525-1528, 2018.

aberrantes. Estudio en 64 cadáveres disecados. Int.J.Morphol., 24(4):5815,2006

Panagouli, E.; Venieratos, D.; Lolis, E. \& Skandalakis, P. Variations in the anatomy of the celiac trunk: A systematic review and clinical implications. Ann. Anat., 195(6):501-511, 2013.

Poynter, C. W. M. Congenital anomalies of the arteries and veins of the human body with bibliography. The University Studies of the University of Nebraska. 22:1-106, 1922.

Hiatt, J. R.; Gabbay, J. \& Busuttil, R. W. Surgical anatomy of the hepatic arteries in 1000 cases. Ann. Surg., 220(1):50-2, 1994.

Suzuki, T.; Nakayasu, A.; Kawabe, K.; Takeda, H. \& Honjo, I. Surgical significance of anatomic variations of the hepatic artery. Am. J. Surg., 122:505-12, 1971.

Tandler, J. Zur entwickelunggeschicte des menschliden darmarterien. Anat. Hefte., 23:189-209 y 475-99, 1906.

En Yi, S.Q.; Terayama, H.; Naito, M.; Hayashi, S.; Moriyama, H.; Tsuchida, A.; Itoh, M. A common celiacomesenteric trunk, and a brief review of the literature. Ann. Anat. 189(5):482-8, 2007.

Tidemann, 1822 apud Michels, N. A. Variational anatomy of the hepatic, cystic, and retroduodenal arteries; a statistical analysis of their origin, distribution, and relations to the biliary ducts in two hundred bodies AMA Arch. Surg., 66(1):20-34, 1953.

Vandamme, J. P. J.; Bonte, J. \& Schueren, G. A revaluation of hepatic and cystic arteries. The importance of the aberrant hepatic branches. Acta Anat., 73:192-209, 1969.

Varotti, G.; Gondolesi, G. E.; Goldman, J.; Wayne, M.; Florman, S. S.; Schwartz, M. E.; Miller, C. M. \& Sukru, E. Anatomic variations in right liver living donors. J. Am. Coll. Surg., 198(4):577-82, 2004.

\section{Dirección para correspondencia. \\ Dra. Bélgica Vásquez \\ Facultad de Ciencias de la Salud \\ Universidad de Tarapacá \\ CHILE}

Email: bvasquez@uta.cl

Recibido: 07-05-2018

Aceptado: 18-09-2018 